Subcategories of JIA included: polyarticular JIA negative Rheumatoid Factor (RF) in $39.77 \%$ of cases, enthesitis-related arthritis in $27.07 \%$ of cases, polyarticular JIA positive RF in $14.36 \%$ of cases, oligoarticular JIA in $14.9 \%$ of cases, systemic JIA in $3.31 \%$ of cases and psoriatic JIA in $0.59 \%$ of patients.

Conclusion: Both the data from the questionnaires, but especially the family drawing tests, suggest that the prolonged state of stress with anxiety, sadness, pain and depression, in combination with starvation, lack of parental love and the genetic predisposition, have contributed to the emergence of chronic arthritis, pathology that is growing more and more in recent years in Romania.

References:

[1] Dube SR, Fairweather D, Pearson WS, Felitti VJ, Anda RF, Croft JB. Cumulative childhood stress and autoimmune disease in adults. Psychosom Med. 2009; 71:243-250.

[2] Song H, Fang F, Tomasson G, et al. Association of stress-related disorders with subsequent autoimmune disease. JAMA, 2018; 319:2388-2400.

[3] Vallerand IA, Patten SB, Barnabe C. Depression and the risk of rheumatoid arthritis. Curr Opin Rheumatol. 2019; 31(3):279-284.

Disclosure of Interests: None declared

DOI: 10.1136/annrheumdis-2020-eular.6118

\section{FRI0639-HPR EFFECTS OF VIBROACOUSTIC THERAPY IN CHRONIC MUSCULOSKELETAL PAIN IN CHILDREN AND ADOLESCENTS}

C. Ailioaie $^{1}$, L. Ailioaie ${ }^{1} .{ }^{1}$ Universitatea Alexandru loan Cuza, Medical Physics, lași, Romania

Background: Musculoskeletal chronic pain in children and adolescents is a major cause of locomotor and mental disabilities, with a significant impact on school activities and social costs. Vibroacoustic therapy is used to reduce stress, pain, anxiety and to increase the well-being of children and adults, in order to relax the body, brain and to relieve the symptoms of the disease. It was proved that sound waves imprint movement in water molecules, accelerate lymph and blood circulation, eliminate toxic metabolic waste, vitalize the "good bacteria" by suppressing the activities of "bad bacteria" and balancing the gut microbiota.

Objectives: Aim of study was to evaluate the effect of vibroacoustic therapy on pain and well-being of patients with chronic musculoskeletal pain.

Methods: Between 2017 and December 2019, 84 patients with a mean age of 11.9 years with chronic pain from different musculoskeletal disorders were studied. Patients were randomly divided into: group I (46 patients) received vibroacoustic therapy and group II (38 patients), control, received placebo vibroacoustic therapy.

All patients and parents/legal guardians were informed about the treatment and signed an informed consent. Inclusion criteria were: diagnosis of the disease by the rheumatologist specialist and the initial pain measured on the Visual Analog Scale (VAS)> 5. Exclusion criteria were: patients with acute inflammatory conditions, mental retardation, psychosis, high blood pressure, internal or external bleeding, pregnancy, hearing loss. Group I (46 patients) received vibroacoustic therapy with a special system, in which the patient listens in the headphones a healing music and at the same time, a therapist posts a special pillow that imprints music vibrations on the painful areas. Patients had a session three times a week, for 20 minutes, with a vibrational frequency of $115 \mathrm{~Hz}$, for 4 weeks, then repeated the protocol after a break of 8 weeks.

Group II (38 patients), control, was treated with conventional drugs and placebo vibroacoustic.

Effects of vibroacoustic therapy were evaluated by the pediatric rheumatologist who completed a questionnaire for the evolution of the functional ability in daily living activities (Child Health Assessment Questionnaire) (scores 0-3; 0 = without disabilities; $3=$ disabled) and the intensity of the child's pain by VAS score $0-10,(0=$ no pain, 10 = severe pain), at the beginning of treatment, after 4 weeks and in the end. Results: In group I, after 12 sessions of the vibroacoustic therapy, VAS pain scores decreased from 8.6 to 4.2 , comparatively to only a decrease from 8.2 to 6.7 in the control group ( $p=0.05$ ); the functional activity score was improved with $47 \%$, comparatively with only $23 \%$ in the control group $(p=0.02)$.

At the end of the study, the VAS pain score improvement in group I was $68 \%$, compared to only $29 \%$ in the control group $(p=0.01)$. All patients who were treated with vibroacustic music had a better functional activity score in the end of the study, significantly better compared to the placebo control group $(p<0.05)$.

Conclusion: Vibroacoustic therapy had positive effects in significantly reducing pain, and especially for the areas of the body where the vibrational cushion was applied, comparatively with control group $(p<0.05)$.

Vibroacoustic therapy is a non-pharmacological, non-invasive, no-side-effect treatment modality which has induced patient relaxation, has reduced muscle tension and improved the range of motion, helping a lot in the process of patient's rehabilitation. Sonic vibrations transmitted by the bone conduction adjust the balance of the autonomic nerves, deeply relax the mind and remove excess body tension.

\section{References:}

[1] Boyd-Brewer C, McCaffrey R. Vibroacoustic Sound Therapy Improves Pain Management and More. Holist Nurs Pract 2004;18(3):111-118.

[2] Kita K. The 4 major effects of Kita sound healing. The Society for Harmonic Science. Accessed at 27.01.2020. https://www.sound-healing.jp/en/the_4 major_effects_of_kita_sound_healing.html

Disclosure of Interests: None declared

DOI: 10.1136/annrheumdis-2020-eular.6290

\section{FRI0640-HPR USEFULNESS OF ISOTOPIC RADIOSYNOVIORTESIS IN RHEUMATIC DISEASES WITH CHRONIC REFRACTORY SYNOVITIS.}

J. Anino-Fernández ${ }^{1}$, A. I. Rebollo Giménez ${ }^{1}$, M. A. Ramírez Huaranga',

D. Castro'. ' Hospital Ciudad Real, Rhematology, Ciudad Real, Spain

Background: Radioactive synoviortesis consists of the intra-articular injection of particles from a radioisotopic colloidal suspension, to achieve the selective destruction of the synovial membrane, respecting the cartilage and bone

Objectives: To describe the experience of performing isotopic radiosynoviortesis (RSO) as an alternative technique to the traditional procedures in the treatment of chronic synovitis refractory in patients of our health area and to define the pathologies, clinical improvement and safety in patients treated with the above described method.

Methods: Observational, descriptive and cross-sectional study by reviewing radiosynoviortesis performed in patients with chronic arthritis refractory between 2005 and 2019 at the General University Hospital of Ciudad Real and comparing to common treatments, which normally consist of corticosteroids, disease-modifying drugs, biological therapy, nonsteroidal anti-inflammatory drugs and opioids. The study group involved 65 patients aged 37 to 75 years that had been diagnosed of rheumatic diseases such as rheumatoid arthritis, spondyloarthropathy, gout, osteoarthritis, villonodular synovitis, lupus and undifferentiated arthritis.

Once medical records were reviewed, and prior to the procedure, the following information was registered into a database: age, sex, pathology, treatment received, infiltrations, affected joint, mobility, radiographic stage, laboratory parameters (VSG and PCR), Health Assessment Questionnaire (HAQ) and visual analog pain scale (VAS). After 6 months of treatment, clinical improvement provided by radiosynoviortesis was evaluated and a second database was created by collecting the following data: physical examination, $\mathrm{HAQ}$, laboratory parameters (VSG and PCR), VAS, mobility and subjective perception of the patient. In addition, short-term complications were also recorded.

After 6 months of radiosynoviortesis treatment, clinical improvement was established as a consequence of the following parameters: absence of inflammation on physical examination, improvement of inflammation reactants in comparison to the beginning of the procedure -assuming a decrease of $\geq 2 \mathrm{~mm}$ in ESR and $\geq 0,2 \mathrm{mg} / \mathrm{dl}$ in CPR as such-, enhancement of $\mathrm{HAQ}$-decrease of $\geq 0.25$ compared to the beginning of the treatment-, EVA improvement determined by a reduction of $>25 \%$ in relation to the initial value and increase of mobility. Additionally, the subjective perception of the patient was also considered as evidence of the. clinical improvement.

Results: on the sixth month of treatment, $58.4 \%$ of the individuals perceived a good clinical improvement, which was qualified as excellent by $6.2 \%$ of them. $56.9 \%$ of patients also presented pain reduction in the VAS scale, together with a decrease in joint swelling in $66 \%$ of the cases and an enhanced joint mobility in $69.2 \%$ of them. No side effects appeared due to treatment. An improvement in $\mathrm{HAQ}$ was observed in $67.7 \%$ of the observations and inflammation parameters (both ESR and CRP) were reduced in $64.6 \%$ of the subjects. The pathologies in which the technique has been most frequently prescribed are villonodular synovitis $(27.7 \%)$ and rheumatoid arthritis $(20 \%)$

Conclusion: Isotopic radiosynoviortesis is a useful and safe therapeutic alter native, that leads to a clinical improvement demonstrated by a reduction of pain and inflammation and increased mobility in those patients with chronic synovitis refractory. Compared to usual treatments, currently available data supports the effectiveness and safety of radiosynoviortesis as an alternative therapy for these patients

References:

[1] Margit Szentesi, Zoltán Nagy, Pal Géher, István Papp. A prospective observational study on the long-term results of 90 Yttrium citrate radiosynoviorthesis of synovitis in osteoarthritis of the knee joint. Eur J Nucl Med Mol Imaging (2019) 46:1633-1641

[2] Zwolak R, Majdan M.Contemporary use of radiosynoviorthesis in chronic polyarthrtitis.Wiad Lek. 2017;70(3 pt 2):677-684.

Disclosure of Interests: None declared

DOI: 10.1136/annrheumdis-2020-eular.1795 